\title{
WAVELET-LIKE ANALYSIS IN THE FREQUENCY-DAMPING DOMAIN FOR MODAL PARAMETERS IDENTIFICATION
}

\author{
Giovanni Totis, Marco Sortino, Sandro Belfio
}

DIEGM, University of Udine, Via delle Scienze 206, Udine I33100, ITALY

\begin{abstract}
Modal analysis and parametric model identification play a fundamental role in many fields, especially for the optimization and vibration control of civil structures and complex mechanical systems. In the last decades these techniques have been frequently applied for the development of innovative cutting tools and CNC machine tools. Although several methodologies are available in literature for parametric model identification, there is still a lack of an effective and robust algorithm. In this paper a new algorithm for automatic identification of a parametric model of a linear dynamic Single Input Single Output system with Multiple Degrees of Freedom is presented. Some recent approaches perform the Wavelet decomposition of the Impulse Response in the time-frequency domain. Here a Wavelet-like decomposition of the Frequency Response in the frequency-damping domain is introduced for vibration modes recognition. Afterwards, advanced statistical approaches are applied for vibration modes selection and model generation. The method was successfully tested on a complicated frequency response characterized by several vibration modes, which was obtained from experimental modal analysis performed on a circular saw blade.
\end{abstract}

Keywords: Modal analysis; Parametric Model; Identification; Wavelet; Statistics; Tooling system; Machine; Mechanics
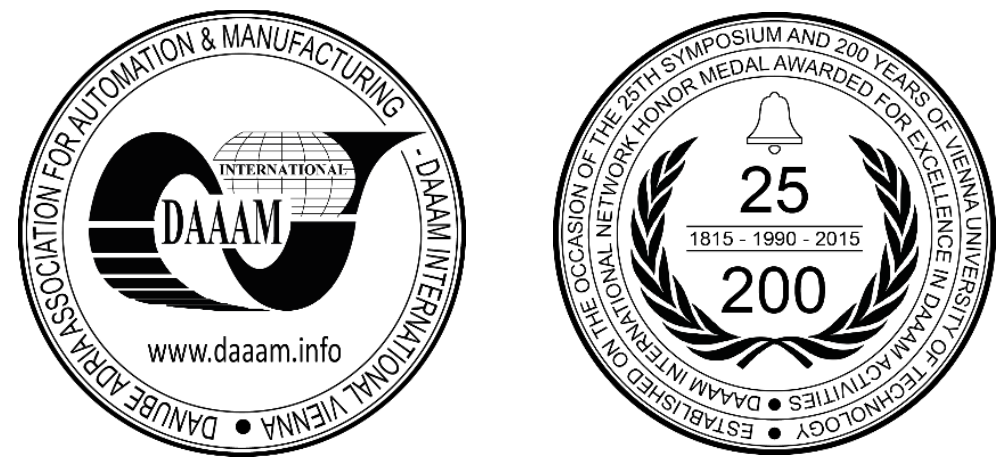

This Publication has to be referred as: Totis, G[iovanni]; Sortino, M[arco] \& Belfio, S[andro] (2016). Wavelet-like Analysis in the Frequency-Damping Domain for Modal Parameters Identification, Proceedings of the 26th DAAAM International Symposium, pp.0580-0588, B. Katalinic (Ed.), Published by DAAAM International, ISBN 978-3-90273407-5, ISSN 1726-9679, Vienna, Austria

DOI: $10.2507 / 26$ th.daaam.proceedings.079 


\section{Introduction}

Nowadays, experimental modal analysis, system identification techniques as well as FE numerical modelling are fundamental tools for design, control, testing and validation of civil structures [1], mechanical components, machines and mechatronic devices. In the last decades, these techniques have been successfully applied for the development of innovative machine tools [2], cutting tools [3] and workpiece fixtures, as well as for the suppression of undesired vibrations occurring during machining processes [4].

The aim of system identification is to determine mathematical models based on a limited number of coefficients/parameters, in order to explain system behaviour with sufficient accuracy in the perspective of design optimization, real-time dynamic control or other practical purposes.

From the seventies, a large number of identification techniques have been conceived [5][6][7][8]. They can be classified as Single Input Single Output (SISO), Single Input Multiple Output (SIMO) or Multiple Input Multiple Output (MIMO) identification techniques, depending on the number of (measured) inputs exciting the system and outputs extracted from it. The most common and effective SISO techniques are illustrated in Table 1. For the sake of completeness, it is important to notice that most of them can be easily extended to SIMO/MIMO systems or they are just special cases of SIMO/MIMO techniques.

\begin{tabular}{|c|c|c|c|c|}
\hline Method (versions and references) & 嶽 & 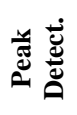 & 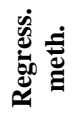 & Highlights \\
\hline $\begin{array}{l}\text { Linear Least Squares Method in the Time } \\
\text { Domain (Prony classical version [8]; ARMA / } \\
\text { ARX / IIR+OE versions [5][9][6]) }\end{array}$ & $\mathrm{T}$ & M & $\mathrm{D}$ & $\begin{array}{l}\text { No control on the nature of poles and zeros; ill conditioned and } \\
\text { sensitive to noise; poor interpolation capabilities in the presence of } \\
\text { MDOF systems. }\end{array}$ \\
\hline $\begin{array}{l}\text { Least Squares Complex Exponential Method } \\
\text { (LSCE) [5][10][7] }\end{array}$ & $\mathrm{T}$ & M & I & $\begin{array}{l}\text { Based on Impulse Response Function (IRF) analysis. Usually ill } \\
\text { conditioned and very sensitive to noise. }\end{array}$ \\
\hline $\begin{array}{l}\text { Ibrahim Time Domain Method ([5][11]; Single } \\
\text { Station Time Domain Method (SISO version) } \\
[12][13][14])\end{array}$ & $\mathrm{T}$ & M & I & $\begin{array}{l}\text { Based on the analysis of free response decays - which are } \\
\text { generally noisier than the IRF estimates. Need for little interaction; } \\
\text { effective calculation of closely spaced modes. Tendency to give } \\
\text { non-conservative damping estimates with noisy data. }\end{array}$ \\
\hline $\begin{array}{l}\text { Stochastic Subspace identification Methods } \\
{[15][16][17][18]}\end{array}$ & $\mathrm{T}$ & M & $\mathrm{D}$ & $\begin{array}{l}\text { Derived from control theory and state space approaches. Rather } \\
\text { accurate and reliable; relatively time consuming in comparison to } \\
\text { other methods. }\end{array}$ \\
\hline $\begin{array}{l}\text { Linear Least Squares Method in the Frequency } \\
\text { Domain (Classical Zobel-Levy [8]; Prony } \\
\text { method [5][19]; Rational Fraction Polynomial } \\
[5][20])\end{array}$ & $\mathrm{F}$ & M & $\mathrm{D}$ & $\begin{array}{l}\text { There is no control on the nature of poles and zeros; it is very } \\
\text { difficult to identify MDOF systems with closely spaced modes. }\end{array}$ \\
\hline $\begin{array}{l}\text { Basic Methods in the Frequency Domain (Peak } \\
\text { Picking Amplitude, Quadrature Response/Max. } \\
\text { Quadr. Component, Kennedy-Pancu / Circle- } \\
\text { Fitting [5][7]) }\end{array}$ & $\mathrm{F}$ & $S$ & I & $\begin{array}{l}\text { Very sensitive to measurement noise and to the presence of other } \\
\text { close vibration modes. Automation level is poor. }\end{array}$ \\
\hline $\begin{array}{l}\text { Inverse Method (Bendent / Dobson Method } \\
[21][5][7])\end{array}$ & $\mathrm{F}$ & S & I & $\begin{array}{l}\text { Better than other basic methods in the frequency domain, but still } \\
\text { sensitive to measurement noise and to the presence of other close } \\
\text { vibration modes. }\end{array}$ \\
\hline Ewins-Gleeson method [5][22] & $\mathrm{F}$ & M & I & $\begin{array}{l}\text { Effective only for lightly damped structures; sensitive to the points } \\
\text { chosen by the user from the FRFs, especially with noisy } \\
\text { measurements. }\end{array}$ \\
\hline Wavelet-based identification methods [23-27] & $\mathrm{B}$ & M & I & $\begin{array}{l}\text { Promising thank to the time-frequency filtering properties of } \\
\text { wavelets. Possibly sensitive to noise, they may have some } \\
\text { problems in the presence of highly damped or closely spaced } \\
\text { modes. They may be time-consuming. }\end{array}$ \\
\hline
\end{tabular}

T=Time; F=Frequency; B=Time-Frequency; S=Single; M=Multiple; D=Direct; I=Indirect.

Table 1. Main identification techniques for Single Input Single Output linear dynamic systems with Multiple Degrees of Freedom

Identification methods can be split into three broad categories, depending on the domain where the identification procedure is carried out: time domain methods, frequency domain methods and time-frequency domain methods.

As reported by several authors [25][16][1], identification techniques in the frequency domain are usually more vulnerable to measurement noise than the time domain methods and they may be rather inaccurate, especially in the case of highly damped systems with severe modal interference. In addition, many of them (for instance, the GaukrogerSkingle-Heron (GSH) method [28], the Ewins-Gleeson method [22] and other basic frequency domain methods [5]) are affected by a low level of automation, i.e. their performance is strongly dependent on the choices and expertise of the user.

At the other side, time domain methods frequently provide accurate results if the measured responses are not severely disturbed by noise. Among the different time domain techniques, the Stochastic Subspace Identification approach 
seems to be the most accurate and insensitive to measurement noise, nevertheless it may require longer computation time than other methods [16][1].

Basically, vibration mode detection can be performed in two different ways: each vibration mode can be analysed and identified separately - single peak detection approach - or all the vibration modes are identified concurrently multiple peak detection approach. When working in the time domain, the multiple peak detection is typically adopted since it is very difficult to isolate the effect of a single mode.

Let us now consider a SISO linear dynamic system with Multiple Degrees Of Freedom (MDOF), such as a vibrating mechanical structure stressed by a force $\mathrm{F}$ (applied at a fixed point along a fixed direction) and oscillating with vibration $\mathrm{u}$ (at a given inspection point and along a given direction). Under these hypotheses, the model Wmod approximating the experimental frequency response Wexp is usually expressed by a rational polynomial in the frequency domain, that is

$$
W_{\text {exp }}(j \omega)=\frac{u(j \omega)}{F(j \omega)} \approx W_{\text {mod }}(j \omega)=\frac{\sum_{r=0}^{Z} b_{r}(j \omega)^{r}}{\sum_{k=0}^{P} a_{k}(j \omega)^{k}}
$$

where the coefficients $b_{r}$ and $a_{k}$ have to be determined through the identification procedure. Usually, the number of zeros $\mathrm{Z}$ appearing in Equation (1) is strictly smaller than the number of poles P, representing model order.

Alternatively, the model can be expressed as a linear superposition of simple harmonic oscillators, as follows

$$
W_{\text {mod }}(j \omega)=\sum_{h=1}^{M} \frac{G_{h}}{\left(j \omega / \omega_{n, h}\right)^{2}+2 \xi_{h} \omega_{n, h}\left(j \omega / \omega_{n, h}\right)+1}
$$

where $G_{h}$ is the static compliance, $\omega_{n, h}$ is the natural pulsation and $\xi_{h}$ is the damping coefficient of the $h^{\text {th }}$ harmonic oscillator, and $M$ is the total number of vibration modes composing the model. When Equation (2) is assumed, $M$ pairs of complex conjugate poles are present. In general, the last model formulation is strongly preferred since it is derived from well-known physical principles governing the dynamic behavior of vibrating mechanical structures. When coefficients of Equation (1) are the unknowns of the linear regression performed by the identification procedure, the algorithm is classified as "direct". Otherwise, when the modal parameters of Equation (2) are the target unknowns, the algorithm is classified as "indirect". Indirect algorithms are generally more effective and reliable, since they are less sensitive to measurement noise. For instance, direct algorithms such as the Linear Least Squares Method in the Time Domain and the Linear Least Squares Method in the Frequency Domain may easily identify unstable poles or anomalous zeros with positive real parts. On the contrary, these problems may rarely arise when using indirect algorithms, because the physically based model structure of Equation (2) is assumed from the beginning.

A common problem affecting identification algorithms is model order selection. In most cases, model order is usually unknown. Moreover, some false vibration modes improving mathematical interpolation of noisy experimental data can be recognized as structural modes, although they have no physical meaning. The classical tool for model selection is the stabilization chart, where the characteristics of the obtained modes are analysed by increasing model order [7][18]. Other powerful techniques are only available in case of SIMO/MIMO identification algorithms, such as the Modal Assurance Criterion (MAC), which is based on the comparison between the mode eigenshapes obtained from identification and those simulated through FE methods [7]. Specific techniques such as the random decrement technique [29] or the approach proposed by Mohanty et al. in [13] can be applied for removing undesired harmonics from experimental data, especially when performing Operational Modal Analysis, i.e. when the input excitation forces are unknown or cannot be observed.

In recent years, some new techniques for model identification operating in the time-frequency domain have been developed. They are based on the analysis of the Impulse Response through different kinds of discrete and continuous Wavelet Transforms [23-27]. The success of these approaches relies in the intrinsic capability of the Wavelet Transform of analysing non-stationary signals, hence their effectiveness for mode decoupling (even when there is a strong dynamic interference) and for noise suppression.

In this paper, an original SISO identification technique is presented for MDOF linear dynamic systems expressed in the structural form of Equation (2). The core of the algorithm was inspired by the Wavelet decomposition methodology. Despite other methods found in literature - which analyse the Impulse Response in the conventional time-frequency domain thorough the Wavelet Transform - here a Wavelet-like decomposition of the Frequency Response is carried out in the frequency-damping domain. By so doing, the main vibration modes composing the Frequency Response can be recognized. Afterwards, advanced statistical tools such as the linear stepwise regression are applied for mode selection and for model generation. 


\section{Wavelet-inspired algorithm for modal parameters identification}

At the beginning, none of the harmonic oscillators composing the model frequency response $W_{\text {mod }}$ of Equation (2) are known. The only available information is the experimental frequency response, provided in a non-parametric form called Empirical Transfer Function Estimate (ETFE) which is basically a Discrete Fourier Transform representing the average dynamic behavior of the system in the frequency domain.

The proposed algorithm is capable to detect all the vibrations modes step by step by applying the following procedure. Let us split the model frequency response in two parts, the (unknown) generic $k^{\text {th }}$ harmonic oscillator and the (unknown) residual part $W_{\text {res }}$, as follows:

$$
W_{\text {mod }}(j \omega)=G_{k} \underbrace{\left[\frac{1}{\left(j \omega / \omega_{n, k}\right)^{2}+2 \xi_{k} \omega_{n, k}\left(j \omega / \omega_{n, k}\right)+1}\right]}_{w_{k}(j \omega)}+\underbrace{\sum_{h \neq k} \frac{G_{h}}{\left(j \omega / \omega_{n, h}\right)^{2}+2 \xi_{h} \omega_{n, h}\left(j \omega / \omega_{n, h}\right)+1}}_{W_{\text {res }}(j \omega)}
$$

where the $k^{\text {th }}$ mode is further decomposed as the product of $w_{k}$ (which does only depend on the natural pulsation $\omega_{n, k}$ and on the damping coefficient $\xi_{k}$ but in a strongly non-linear way) and the static compliance $G_{k}$. Let us fix the values of both $\omega_{n, k}$ and $\xi_{k}$ while keeping $G_{k}$ as a free parameter. Let us approximate the residual part $W_{\text {res }}$ by Taylor expansion in the neighborhood of the natural pulsation $\omega_{n, k}$, as follows

$$
\underbrace{W_{\text {exp }}(j \omega)}_{\in \mathbb{C}} \approx \underbrace{W_{\text {mod }}(j \omega)}_{\in \mathbb{C}} \cong \underbrace{G_{k}}_{\in \in \mathbb{R}} \underbrace{w_{k}(j \omega)}_{\in \mathbb{C}}+\underbrace{W_{\text {res }}\left(\omega_{n, k}\right)}_{\in \mathbb{C}}+\underbrace{\left.\frac{\partial W_{\text {res }}}{\partial \omega}\right|_{\left(\omega_{n, k}\right)}}_{\in \mathbb{C}} \underbrace{\left(\omega-\omega_{n, k}\right)}_{\in \mathbb{R}} \quad \omega \approx \omega_{n, k}
$$

In order to simplify notation, let us define

$$
\left\{\begin{array}{l}
R_{\omega, \exp }=\operatorname{Re}\left\{W_{\text {exp }}(j \omega)\right\} \\
I_{\omega, \exp }=\operatorname{Im}\left\{W_{\text {exp }}(j \omega)\right\} \\
R_{\omega, k}=\operatorname{Re}\left\{w_{k}(j \omega)\right\} \\
I_{\omega, k}=\operatorname{Im}\left\{w_{k}(j \omega)\right\}
\end{array}\right.
$$

After some algebraic manipulations and after separation of the real from the imaginary part of Equation (4) one obtains

$$
\left\{\begin{array}{l}
R_{\omega, \exp } \approx G_{k} R_{\omega, k}+a_{0}+a_{1} \omega \\
I_{\omega, \exp } \approx G_{k} I_{\omega, k}+b_{0}+b_{1} \omega
\end{array} \quad \omega \approx \omega_{n, k}\right.
$$

where $a_{0}, a_{1}, b_{0}, b_{1}$ are real constants depending on the natural pulsation $\omega_{n, k}$. The frequency bandwidth which is strongly influenced by the $k^{\text {th }}$ harmonic oscillator can be expressed by

$$
\omega \in\left[\omega_{n, k}\left(1-\alpha \xi_{k}\right), \omega_{n, k}\left(1+\alpha \xi_{k}\right)\right]
$$

where the constant $\alpha$ was set to 3 after some preliminary analysis. As expected, the collocation of the harmonic oscillator depends on its natural pulsation, while the bandwidth width depends on damping. In other words, natural pulsation acts as the time localization parameter while damping acts as the scale parameter influencing Mother Wavelet in the conventional Wavelet approach.

It is finally possible to assemble a linear system by considering all the discrete frequencies $\omega_{1}, \ldots, \omega_{q}$ composing the experimental frequency response which are within the frequency bandwidth (7). The result is

$$
\left[\begin{array}{c}
R_{\omega 1, \exp } \\
\vdots \\
R_{\omega q, \exp } \\
I_{\omega 1, \exp } \\
\vdots \\
I_{\omega q, \exp }
\end{array}\right] \approx\left[\begin{array}{ccccc}
R_{\omega 1, k} & 1 & \omega_{1} & 0 & 0 \\
\vdots & \vdots & \vdots & \vdots & \vdots \\
R_{\omega q, k} & 1 & \omega_{q} & 0 & 0 \\
I_{\omega 1, k} & 0 & 0 & 1 & \omega_{1} \\
\vdots & \vdots & \vdots & \vdots & \vdots \\
I_{\omega q, k} & 0 & 0 & 1 & \omega_{q}
\end{array}\right]\left[\begin{array}{c}
G_{k} \\
a_{0} \\
a_{1} \\
b_{0} \\
b_{1}
\end{array}\right]
$$

The unknown coefficients of Equation (8) are determined by multiple linear regression. Afterwards, the real and imaginary parts can be separated, in order to compute the vectors 
$\boldsymbol{R}_{k, \text { exp }}=\left[\begin{array}{c}R_{\omega 1, \text { exp }} \\ \vdots \\ R_{\omega q, \text { exp }}\end{array}\right]-\left[\begin{array}{cc}1 & \omega_{1} \\ \vdots & \vdots \\ 1 & \omega_{q}\end{array}\right]\left[\begin{array}{l}a_{0} \\ a_{1}\end{array}\right] \approx\left[\begin{array}{c}R_{\omega 1, k} \\ \vdots \\ R_{\omega q, k}\end{array}\right] G_{k}=\boldsymbol{R}_{k, \bmod }$

and similarly

$$
\boldsymbol{I}_{k, \exp }=\left[\begin{array}{c}
I_{\omega 1, \exp } \\
\vdots \\
I_{\omega q, \exp }
\end{array}\right]-\left[\begin{array}{cc}
1 & \omega_{1} \\
\vdots & \vdots \\
1 & \omega_{q}
\end{array}\right]\left[\begin{array}{l}
b_{0} \\
b_{1}
\end{array}\right] \approx\left[\begin{array}{c}
I_{\omega 1, k} \\
\vdots \\
I_{\omega q, k}
\end{array}\right] G_{k}=\boldsymbol{I}_{k, \bmod }
$$

These four vectors are finally used to determine the degree of correlation between the $k^{\text {th }}$ harmonic oscillator - having natural pulsation $\omega_{n, k}$ and damping coefficient $\xi_{k}$-and the experimental frequency response purified from the contribution of all the other oscillators. Specifically, the following indicator was introduced:

$$
\Delta R_{k}^{2}=\frac{1}{2}\left[R^{2}\left(\boldsymbol{R}_{k, \text { exp }}, \boldsymbol{R}_{k, \text { mod }}\right)+R^{2}\left(\boldsymbol{I}_{k, \text { exp }}, \boldsymbol{I}_{k, \text { mod }}\right)\right], \quad 0 \leq \Delta R_{k}^{2} \leq 1
$$

which has demonstrated to be very effective for mode identification.

The aforesaid procedure is then iterated by varying the combination of modal parameters $(\omega n, k, \xi k)$ within an admissible region

$$
\left(\omega_{n, k}, \xi_{k}\right) \in D=\left[\omega_{\min }, \omega_{\max }\right] \times\left[\xi_{\min }, \xi_{\max }\right]
$$

which has to be preliminarily defined by taking into account the quality of the available experimental data and the typical characteristics of the system under examination. For instance, the coherence function associated to the measured frequency response will limit the admissible frequency bandwidth.

Before starting computation, domain (12) is split into an uneven grid, according to some rules derived from physical and numerical observations.

First, for a given damping coefficient the frequency interval should be evenly discretized in logarithmic scale. This implies a non-uniform subdivision in natural scale, with increasing subinterval amplitude

$$
\Delta \omega_{n, k}=\omega_{n, k+1}-\omega_{n, k}=\beta \xi_{k} \omega_{n, k}
$$

Similarly, the damping coefficient interval should be evenly discretized in logarithmic scale, again implying an increasing subinterval amplitude

$$
\Delta \xi_{k}=\xi_{k+1}-\xi_{k}=\gamma \xi_{k}
$$

As a rule of thumb, grid parameter $\beta$ should be smaller than $1 / 4$ while $\gamma$ should be smaller than $1 / 10$. However, they can be further refined by trial and error, until a satisfactory interpolation result is achieved.

After computation, the map $\Delta R_{k}^{2}\left(\omega_{n, k}, \xi_{k}\right)$ is available and can be analyzed for the automatic and robust selection of the most important vibration modes composing the experimental frequency response.

The best candidates are indeed the points $\left(\omega_{n, k}, \xi_{k}\right)$ corresponding to local maxima of the $\Delta R_{k}^{2}$ function. They can be found within the closed regions of the $\left(\omega_{n, k}, \xi_{k}\right)$ plane characterized by

$$
\Delta R_{k}^{2}\left(\omega_{n, k}, \xi_{k}\right) \geq \eta
$$

When there is a good signal to noise ratio, $\eta$ can be set around 0.9. However, this parameter may need some adjustment when experimental data are contaminated by considerable noise.

For each mode and for each pulsation within the frequency range of interest, the real and imaginary parts of the wk oscillators are computed. Eventually, Equation (2) is rewritten as the linear system

$$
\left[\begin{array}{c}
R_{\omega 1, \exp } \\
\vdots \\
R_{\omega q, \exp } \\
I_{\omega 1, \exp } \\
\vdots \\
I_{\omega q, \exp }
\end{array}\right] \approx\left[\begin{array}{cccc}
R_{\omega 1,1} & R_{\omega 1,2} & \cdots & R_{\omega 1, M} \\
\vdots & \vdots & & \vdots \\
R_{\omega q, 1} & R_{\omega q, 2} & \cdots & R_{\omega q, M} \\
I_{\omega 1,1} & I_{\omega 1,2} & \cdots & I_{\omega 1, M} \\
\vdots & \vdots & & \vdots \\
I_{\omega q, 1} & I_{\omega q, 2} & \cdots & I_{\omega q, M}
\end{array}\right]\left[\begin{array}{c}
G_{1} \\
G_{2} \\
\vdots \\
G_{M}
\end{array}\right], \quad \omega_{1} \geq \omega_{\min }, \quad \omega_{q} \leq \omega_{\max }
$$


The system (16) is analyzed by the stepwise regression algorithm [30], which is able to recognize the most energetic physical modes while concurrently eliminating other modes. Neglected modes are excluded by the algorithm since they do not give a significant contribution to explain the variability of the experimental frequency response.

The following criterion is then applied for further screening of the candidate modes:

$$
\text { [mode }\left(\omega_{n, k}, \xi_{k}, G_{k}\right) \text { is kept by stepwise regression] AND } \quad\left[\left|G_{k}\right| \geq v G_{t o t, e x p} \text {, with } v \geq 0.1 \%\right]
$$

The second condition can also be written in order to keep only strictly positive or strictly negative static compliances, when their sign is well known from physical considerations. For instance, all static compliances should be strictly positive when considering a SISO vibrating mechanical system with one force input and one vibration output located at the same point, parallel and equally oriented, since all eigenmodes will be locally deformed in the same direction of the input force. This screening phase should be repeated at least once in order to determine a minimal set of significant harmonic oscillators. After eliminating the negligible modes, the last step of the algorithm can be accomplished in order to determine the final model. This can be achieved by performing a weighted linear regression on the selected vibration modes. For instance, higher weights can be assigned in the low-frequency range and in the neighborhood of the resonance frequencies, by also taking into account the behavior of the coherence function. By so doing, both the total static compliance and the resonance peaks will be well interpolated.

It has to be pointed out that model order determination is automatically accomplished by the previous steps, without the need of other tools such as the stabilization diagrams.

\section{Application to a case study}

In order to evaluate the effectiveness of the proposed approach, it was applied to a complicated frequency response derived from modal analysis measurements performed on a circular blade. The blade under examination is used for wood saw cutting.

Transverse vibrations of circular blades during the cutting process may significantly influence the cutting conditions of the engaged teeth, thus they may strongly affect part quality and tool life or they may even hinder process feasibility. For this reason, model identification plays a crucial role in this field, in the perspective of developing better circular blades and for cutting process optimization.

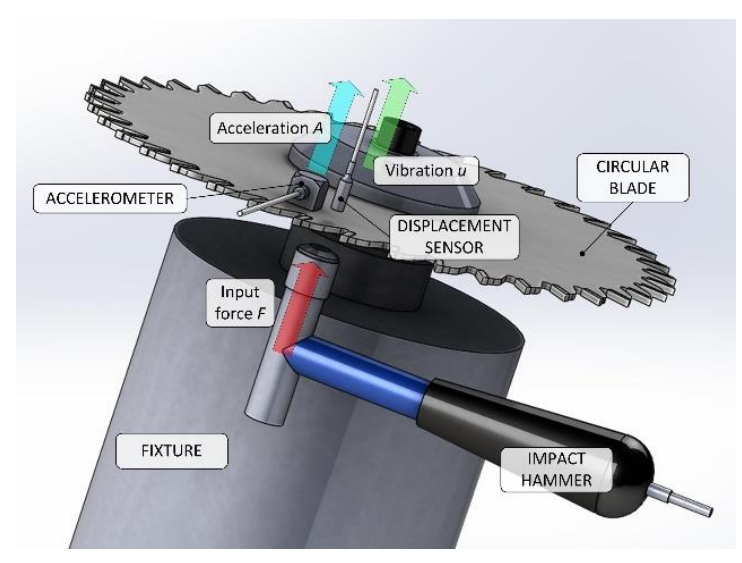

Fig. 1. Schematic picture describing the experimental setup for modal analysis on a circular blade by means of pulse testing technique

The transverse dynamic compliance of a circular blade can be estimated by performing modal analysis in steady i.e. non-rotating configuration, although the real dynamics may slightly depend on spindle rotational speed due to gyroscopic and centrifugal effects. For the sake of simplicity, here only the steady configuration was considered. In detail, a circular blade made of alloy steel, with external diameter $D_{\text {ext }}$ of about $260 \mathrm{~mm}$, uniform thickness $s$ of about $2 \mathrm{~mm}$, number of teeth $z_{t}=40$ was clamped on a special testing flange with external diameter $D_{\text {fla }}$ of about 85 mm, similarly to Figure 1.

Input force $F$ was applied on blade periphery perpendicularly to blade plane by using an impact hammer Dytran type 5800B4, with sensitivity of $2.41 \mathrm{mV} / \mathrm{N}$, connected to an amplifier Kistler type 5134B. Blade vibration was measured by a non-contact eddy current probe Micro-Epsilon type ES1 (measuring range $1 \mathrm{~mm}$, sensitivity $\approx 10 \mathrm{mV} / \mu \mathrm{m}$ ) connected to eddy NCDT 3010-M controller. At the same time, the transversal blade acceleration - at approximately the same inspection point - was detected by a triaxial piezoelectric accelerometer Kistler $8763 \mathrm{~B} 100$ (sensitivity $\approx 50 \mathrm{mV} / \mathrm{g}$ ), connected to the same amplifier used for the impact hammer. Regarding data acquisition, all sensor signals were sampled at $20 \mathrm{kHz}$ by using a National Instruments device (cDAQ-9178 with NI 9215 modules) connected via USB to a PC. Data were elaborated in MathWorks MATLAB environment. 
The Empirical Transfer Function Estimate - ETFE - is illustrated in Figure 2(a), together with the corresponding coherence function. The accelerometer signal was taken as a reference for transfer function computation. The displacement probe signal was mainly used for comparison and correction of the obtained frequency response in the very low frequency range, where the accelerometer output is affect by a bad signal to noise ratio.

The experimental frequency response is characterized by considerable resonance peaks above $200 \mathrm{~Hz}$ which are fairly well in accordance with basic FEM simulations of blade structural dynamics. Minor resonances located in the low-frequency range are likely due to the dynamic interaction between the blade and the fixture device.

By applying the wavelet-like approach described in the previous section (Equations 3-15), the $\Delta R_{k}^{2}$ map of Figure 2(b) was obtained. In the map, several regions with a high degree of correlation are visible. Precisely, 18 local maxima were found with $\Delta R_{k}{ }^{2}>0.9$ within the frequency range of interest $20-1500 \mathrm{~Hz}$. After the screening phase carried out by the stepwise regression algorithm and by the other criterion of Equation (17), only 12 vibration modes were kept, which are listed in Table 2. Accordingly, the final model was built on a minimum number of significant vibration modes, which dominate the experimental measurement. It can be noticed that very close vibration modes were correctly recognized, such as the three modes located around $228 \mathrm{~Hz}$. It has to be recalled that several state of the art algorithms tend to fail in the presence of very closed vibration modes [5].
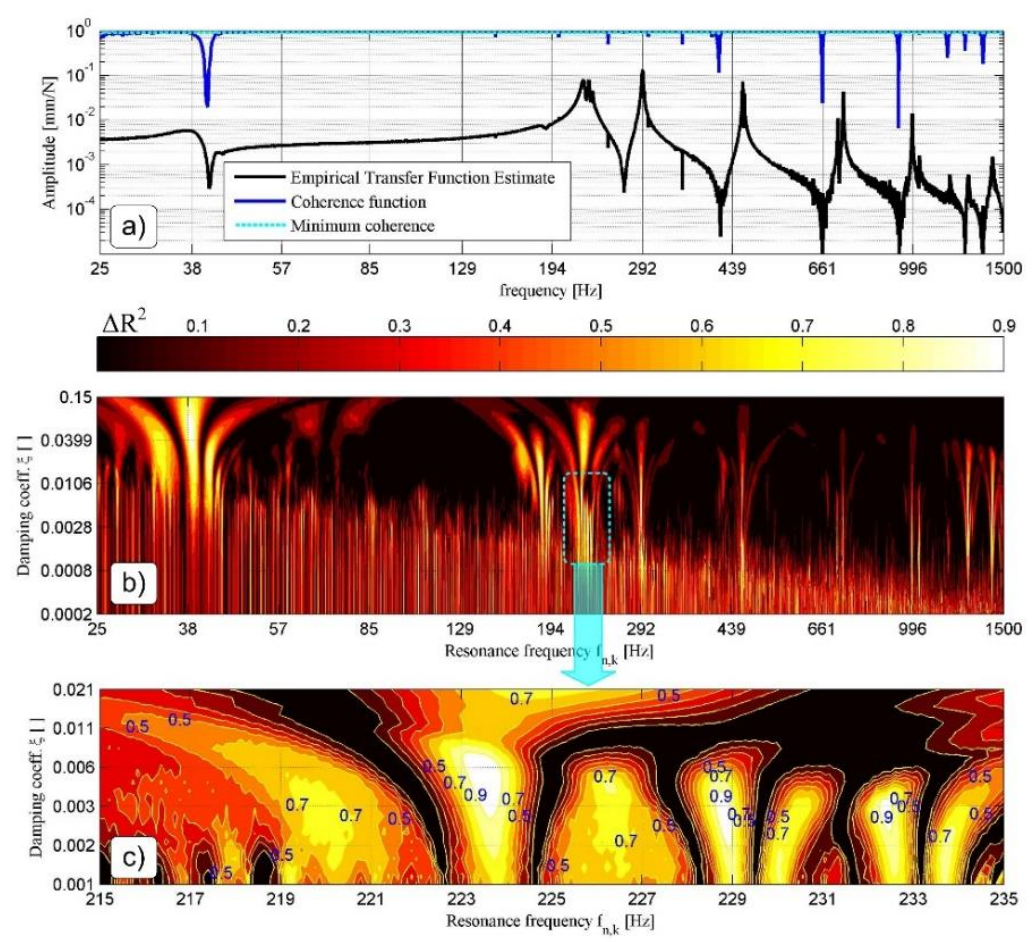

Fig. 2. (a): Amplitude of the Empirical Transfer Function Estimate - ETFE - derived from experimental modal analysis on the circular blade. (b)-(c): Identification of single harmonic oscillators which are most correlated with the experimental frequency response (ETFE) by means of the innovative Wavelet-inspired transformation

Model and experimental transfer function are compared in Figure 3, showing an excellent qualitative agreement between all the considered quantities. In order to test quantitatively model adequacy, the squared correlation coefficient $R^{2}$ between the model and the experimental counterpart was calculated by considering the whole frequency range of interest. For this purpose, the real and imaginary parts were compared separately. For both of them an outstanding value of $R^{2}$ greater than 0.968 was obtained.

In order to further test model adequacy, the relative error between model and experimental frequency response was computed as follows

$$
E_{\text {rel }}(j \omega)=\left|\frac{W_{\text {mod }}(j \omega)-W_{\text {exp }}(j \omega)}{W_{\text {exp }}(j \omega)}\right| \quad[\%]
$$

A weighted average of this error was computed, that is

$$
\mu_{E, r e l}=\frac{\int E_{r e l}(j \omega)\left|W_{e x p}(j \omega)\right| d \omega}{\int\left|W_{e x p}(j \omega)\right| d \omega}
$$


By so doing, the relative error affecting the resonance peaks is enhanced while concurrently reducing the importance of the frequency intervals where the experimental response is very small. In the current case, a satisfactory weighted average of $16.3 \%$ was obtained, proving the effectiveness of the proposed algorithm.

\begin{tabular}{|c|c|c|c|c|c|c|c|c|c|c|c|c|}
\hline Mode \# & 1 & 2 & 3 & 4 & 5 & 6 & 7 & 8 & 9 & 10 & 11 & 12 \\
\hline Nat. freq. $\omega_{n, k}[\mathrm{~Hz}]$ & 38.1 & 185.8 & 223.3 & 228.6 & 232.3 & 291.9 & 459.9 & 462.9 & 707.6 & 725.3 & 991.1 & 1425.7 \\
\hline Damping $\xi_{k}[]$ & $1.04 \mathrm{E}-01$ & $1.21 \mathrm{E}-02$ & $5.48 \mathrm{E}-03$ & $3.23 \mathrm{E}-03$ & $3.23 \mathrm{E}-03$ & $1.90 \mathrm{E}-03$ & $9.80 \mathrm{E}-04$ & $8.58 \mathrm{E}-04$ & $5.77 \mathrm{E}-04$ & $6.59 \mathrm{E}-04$ & $5.77 \mathrm{E}-04$ & $2.48 \mathrm{E}-03$ \\
\hline $\begin{array}{l}\text { Static compl. } G_{k} \\
{[\mu \mathrm{m} / \mathrm{N}]}\end{array}$ & $5.65 \mathrm{E}-01$ & $9.72 \mathrm{E}-02$ & $9.34 \mathrm{E}-01$ & $4.80 \mathrm{E}-01$ & $1.83 \mathrm{E}-01$ & $5.40 \mathrm{E}-01$ & $1.41 \mathrm{E}-01$ & $5.78 \mathrm{E}-02$ & $1.24 \mathrm{E}-02$ & $5.89 \mathrm{E}-02$ & $1.67 \mathrm{E}-02$ & $3.12 \mathrm{E}-03$ \\
\hline $\begin{array}{l}\text { Rel. compl. } G_{k} / G_{t o t, e x p} \\
{[\%]}\end{array}$ & 18.3 & 3.1 & 30.2 & 15.5 & 5.9 & 17.5 & 4.6 & 1.9 & 0.4 & 1.9 & 0.5 & 0.1 \\
\hline
\end{tabular}

Table 2. List of the vibration modes composing the final model
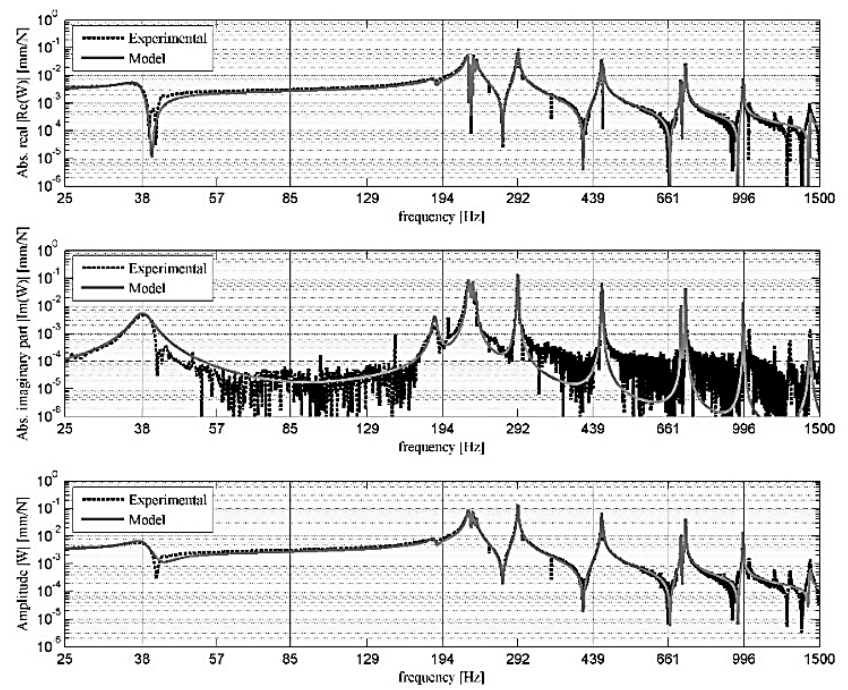

Fig. 3. Comparison between experimental frequency response (ETFE) and the obtained parametric model determined through the identification procedure

\section{Conclusions}

In this paper an original algorithm for automatic and robust identification of a linear SISO MDOF dynamic model of an experimental frequency response was presented. One key hypothesis was that the experimental frequency response can be decomposed into a sum of simple harmonic oscillators, which is usually the case when considering mechanical vibrating systems.

The algorithm consists in two phases: modes identification and modes selection. In the first phase, a waveletinspired approach is used for the identification in the frequency-damping domain of some promising candidates among all possible simple harmonic oscillators. In the second phase, a robust screening is performed by applying some statistical criteria.

The algorithm was successfully tested on a complicated experimental frequency response representing the transversal dynamic behavior of a circular blade clamped by a stiff fixture. The obtained model was capable of representing the static compliance and the resonance peaks with good accuracy. Actually, the interpolation was satisfactory in the whole frequency range of interest. Moreover, very close resonance peaks were correctly recognized.

It would be of further interest to strictly compare the proposed approach with other state of the art algorithms in terms of accuracy, computational time and automation level, also by considering other experimental case studies.

\section{References}

[1] F. Magalhaes, A. Cunha, E. Caetano, R. Brincker, Damping estimation using free decays and ambient vibration tests, Mechanical Systems and Signal Processing, 24 (2010) 1274-1290.

[2] Y. Altintas, C. Brecher, M. Weck, S. Witt, Virtual machine Tool, CIRP Annals - Manufacturing Technology, Volume 54, Issue 2, 2005, pp.115-138.

[3] P. Albertelli, M. Goletti, M. Monno, A new receptance coupling substructure analysis methodology to improve chatter free cutting conditions prediction, International Journal of Machine Tools \& Manufacture, 72 (2013) 16-24.

[4] G. Totis, RCPM - A new method for robust chatter prediction in milling, International Journal of Machine Tools \& Manufacture, 49 (2009) 273-284. 
[5] N. Maia, Extraction of valid modal properties from measured data in structural vibration, Ph.D. Thesis, Imperial College of Science, Technology and Medicine, 1988, London.

[6] L. Ljung, System Identification: Theory for the user, $2^{\text {nd }}$ edition, 1999, Prentice-Hall PTR.

[7] D. J. Ewins, Modal testing: theory, practice and application, $2^{\text {nd }}$ edition, 2000, Research Studies Press LTD, England

[8] R. Isermann, M. Münchhof, Identification of Dynamic Systems, An Introduction with Applications, 2011, SpringerVerlag Berlin Heidelberg.

[9] W. Gersch, R. S.-Z. Liu, Time Series Methods For The Synthesis Of Random Vibration Systems, Trans. of the ASME, Journal of Appl. Mech., Vol. 43/1, 1976, pp. 159-165.

[10] F. R. Spitznogle, A. H. Quazi, Representation And Analysis Of Time-Limited Signals Using A Complex Exponential Algorithm, The Journal of The Acoustical Society of America, Vol. 47, No. 5 (Part 1), 1970, pp. 11501155 .

[11] S. R. Ibrahim, E. C. Mikulcik, A Method for the Direct Identification of Vibration Parameters from the Free Response, The Shock \& Vibration Bulletin, Vol. 47 (4), 1977, pp. 183-198.

[12] S. A. Zaghlool, Single-Station Time-Domain (SSTD) Vibration Testing Technique: Theory And Application, Journal of Sound \& Vibration, 72 (2), 1980, pp. 205-234.

[13] P. Mohanty, D. J. Rixen, Modified SSTD method to account for harmonic excitations during operational modal analysis, Mechanism and Machine Theory, Vol. 39, 2004, pp. 1247-1255.

[14] A. Malekjafarian, R. Brincker, M.R. Ashory, M.M. Khitibi, Identification of closely spaced modes using Ibrahim Time Domain method, Proceedings of the fourth International Operational Modal Analysis Conference, 2010, Istanbul.

[15] V. P. Overschee, B. De Moor, Subspace Identification for Linear Systems, 1996, Kluwer Academic Publisher.

[16] J.-H. Yi, C.-B. Yun, Comparative study on modal identification methods using output-only information, Structural Engineering and Mechanics, Vol.17, 2004, pp.445-466.

[17] P. G. Bakir, Automation of the stabilization diagrams for subspace based system identification, Expert Systems with Applications, Vol.38, 2011, pp. 14390-14397.

[18] F. J. Cara, J. Juan, E. Alarcon, E. Reynders, G. De Roeck, Modal contribution and state space order selection in operational modal analysis, Mechanical Systems and Signal Processing, 38 (2013) 276-298.

[19] J. N. Brittingham, E. K. Miller, J. L. Willows, Pole Extraction From Real-Frequency Information, Proc. of XEEE, Vol. 68, No. 2, 1980, pp. 263-273.

[20] M. H. Richardson, D. L. Formenti, Parameter Estimation From Frequency Response Measurements Using Rational Fraction Polynomials, Proc. of IMAC I, Orlando, Florida, 1982, pp. 8-10.

[21] B. J. Dobson, A Straight-Line Technique For Extracting Modal Properties From Frequency Response Data, Mechanical Systems and Signal Processing, Vol. 1/1, 1987

[22] D. J. Ewins, P. T. Gleeson, A Method for Modal Identification of Lightly Damped Structures, Journal of Sound \& Vibration, 84 (1982) 57-79.

[23] J. Lardies, S. Gouttebroze, Identification of modal parameters using wavelet transform, Journal of Mechanical Sciences, Vol.44 (11), 2002, 2263-2283.

[24] T.-P. Le, P. Argoul, Continuous wavelet transform for modal identification Using free decay response, Journal of Sound and Vibration, 277 (2004) 73-100.

[25] C.S. Huang, W.C. Su, Identification of modal parameters of a time invariant linear system by continuous wavelet transformation, Mechanical Systems and Signal Processing, 21 (2007) 1642-1664.

[26] T.-P. Le, P. Paultre, Modal identification based on continuous wavelet transform and ambient excitation tests, Journal of Sound and Vibration, 331 (2012) 2023-2037.

[27] H. Sarparast, M.R. Ashory, M. Hajiazizi, M. Afzali, M.M. Khatibi, Estimation of modal parameters for structurally damped systems using wavelet transform, European Journal of Mechanics A/Solids, 47 (2014) 82-91.

[28] D. R. Gaukroger, C. W. Skingle, K.H. Heron, Numerical Analysis Of Vector Response Loci, Journal of Sound \& Vibration, Vol.29(3), 1973, pp.341-353.

[29] S. V. Modak, Separation of structural modes and harmonic frequencies in Operational Modal Analysis using random decrement, Mechanical Systems and Signal Processing, 41 (2013) 366-379.

[30] S.Weisberg, Applied linear regression, Wiley Series in Probability and Statistics, $3^{\text {rd }}$ edition, 2005, WileyInterscience. 